\title{
Hydrometrocolpos: A Lower Mesodermal Defects Sequence
}

\author{
Vijaya Lakshmi Bodduluri ${ }^{1}$ (i) $\cdot$ Lata Srinivasan $^{2}$
}

Received: 26 October 2015/Accepted: 7 December 2015/Published online: 17 December 2015

(C) Society of Fetal Medicine 2015

\begin{abstract}
Hydrometrocolpos is a rare congenital disorder, which can present prenatally or after birth. It can be isolated or associated with other abnormalities. The abnormalities associated with hydrometrocolpos can be primary or secondary. Primary abnormalities arise from disruption of embryological processes while secondary abnormalities arise from the pressure effects of the distended vagina and uterus on adjacent organs. An analysis of autopsy findings of four cases of hydrometrocolpos at our center has shown a nonrandom association of certain congenital abnormalities. These abnormalities involve structures arising from the infraumbilical intraembryonic mesoderm. A primary developmental failure or secondary disruption of normally formed tissues results in this condition called lower mesodermal defects sequence. Identification of this condition is important in prognostication and counseling parents.
\end{abstract}

Keywords Hydrometrocolpos - Vaginal atresia - Lower mesodermal defects sequence $\cdot$ Intraembryonic mesoderm

\section{Introduction}

Hydrometrocolpos is the distension of the vagina and uterus with sterile fluid caused by vaginal outlet obstruction. It is an uncommon congenital disorder with an

Vijaya Lakshmi Bodduluri

vijaya.lakshmi3@yahoo.com

1 Department of Fetal Medicine, Mediscan Systems, 197 Dr Natesan Road, Mylapore, Chennai 600004, India

2 Department of Perinatal Pathology, Mediscan Systems, Mylapore, Chennai, India incidence of approximately one in 16,000 female births [1]. Vaginal obstruction may result from vaginal atresia, a vaginal septum, imperforate hymen, persistent urogenital sinus or a cloacal anomaly, depending on the timing and nature of the embryological process that is disrupted [2].

Hydrometrocolpos has a varied presentation. It can be diagnosed in utero, at birth, or at any time up to puberty. The prognosis is excellent if it is due to an isolated abnormality such as in imperforate hymen. The mortality is usually due to associated congenital abnormalities which are present in $17-81 \%$ of cases [3].

$\mathrm{We}$, herein, present the autopsy findings of four cases of hydrometrocolpos, performed during the period 2009-2014. Two of these cases had antenatal ultrasound scan at our center and in the other two cases, the fetuses were sent to us for autopsy. In all four cases, the parents had opted for termination of pregnancy. The autopsy findings illustrate the spectrum of abnormalities associated with hydrometrocolpos arising either as primary congenital abnormalities or secondary to pressure effects from the distended vagina and uterus. Identifying the spectrum of associated abnormalities may provide insight into the etiopathogenesis of hydrometrocolpos, which may, in turn, help in deriving prognostic information and in counseling parents.

\section{Report of Cases}

\section{Case I}

A 29-year-old woman, G2 A1 was referred at 21 weeks of gestation for second opinion on single umbilical artery in the fetus. The same was confirmed on ultrasound scan. At a subsequent visit at 27-28 weeks of gestation, a diagnosis 
of hydrometrocolpos was made. The diagnosis was based on the characteristic four-chamber appearance noted on ultrasound scan. This appearance was constituted by a cystic mass, with a midplane septum, present posterior to the bladder and connected to two uterine cavities (Fig. 1) [4]. The right kidney was absent in the right renal fossa. There was ascites and polyhydramnios.

On autopsy, the right kidney was absent and there was an enlarged bladder with a patent urethra (Fig. 2). The lungs were hypoplastic and there was cardiomegaly. The external genitalia were ambiguous and the anus was imperforate. Autopsy confirmed hydrometrocolpos with uterus didelphys bicollis with longitudinal and transverse vaginal septa (Fig. 3).

\section{Case II}

A 21-year-old primigravida was referred at 31 weeks for ultrasound scan for second opinion on bilateral cystic kidneys with the differential diagnosis of lower urinary tract obstruction or an ovarian cyst in the fetus. On ultrasound scan, bilateral echogenic kidneys with hydronephrosis were identified while the bladder was normal. There was sacral agenesis and a thick-walled cystic mass with internal echoes was noted in the fetal abdomen, measuring $5.9 \times 2.5 \mathrm{~cm}$, and was diagnosed as hydrometrocolpos (Fig. 4).

At autopsy there was hydrometrocolpos with vaginal atresia (Fig. 5). Bilateral hydronephrosis was present with bladder outlet obstruction from urethral atresia. The lungs were hypoplastic and there was aberrant origin of the left external carotid artery from the aortic arch. The external genitalia were ambiguous and the anus was imperforate with high anorectal anomaly. A fetogram confirmed sacral dysgenesis.

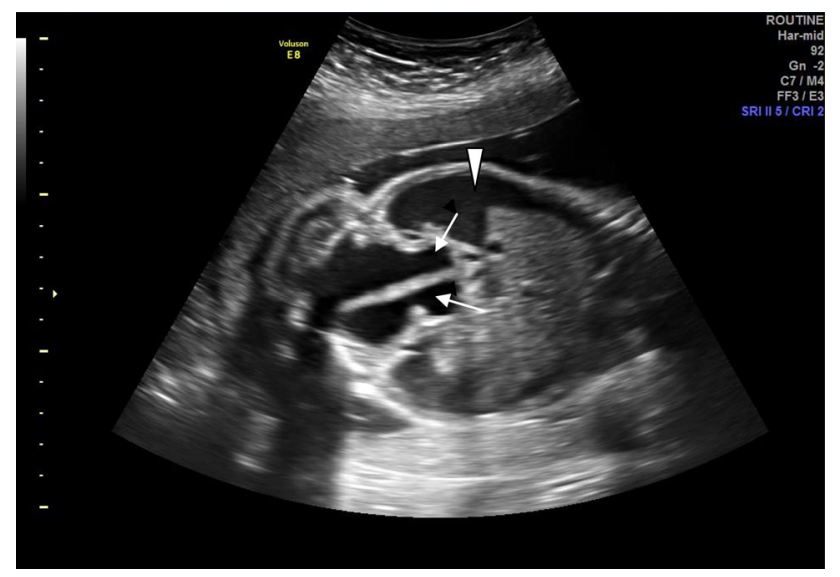

Fig. 1 Sagittal view of the fetal pelvis and abdomen showing a septate cystic mass connected to two dilated uterine cavities (arrows) with the characteristic four-chamber appearance. Also note the presence of fetal ascites (arrowhead)

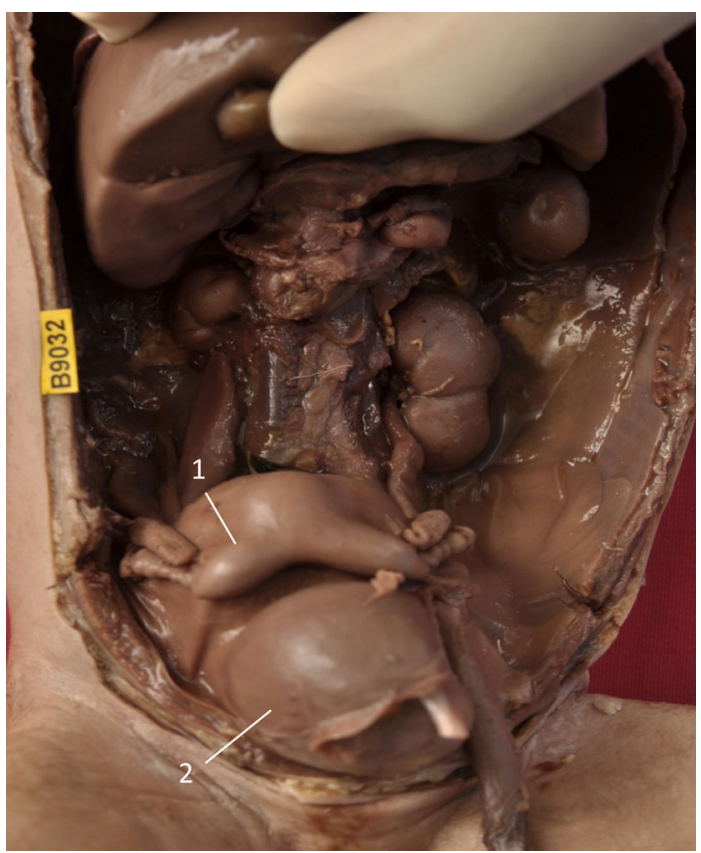

Fig. 2 Autopsy picture showing hydrometrocolpos with uterus didelphys (1) and distended bladder (2)

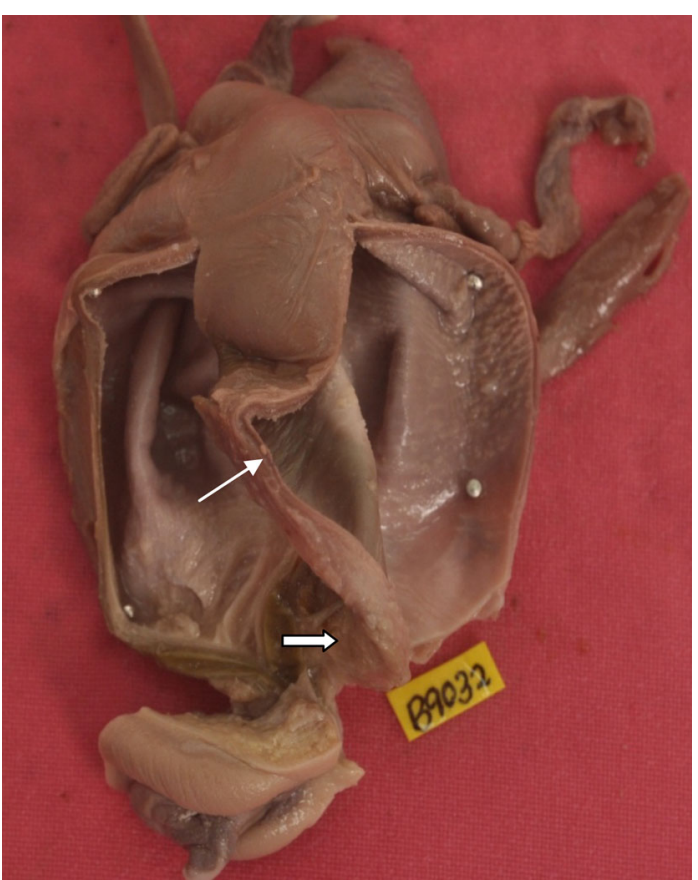

Fig. 3 Cut section of distended vagina showing longitudinal (thin arrow) and transverse (thick arrow) vaginal septa

\section{Case III}

The fetus of a 20 -year-old primigravida at 28 weeks of gestation with an antenatal diagnosis of intrauterine growth restriction and nonimmune hydrops fetalis was sent for 


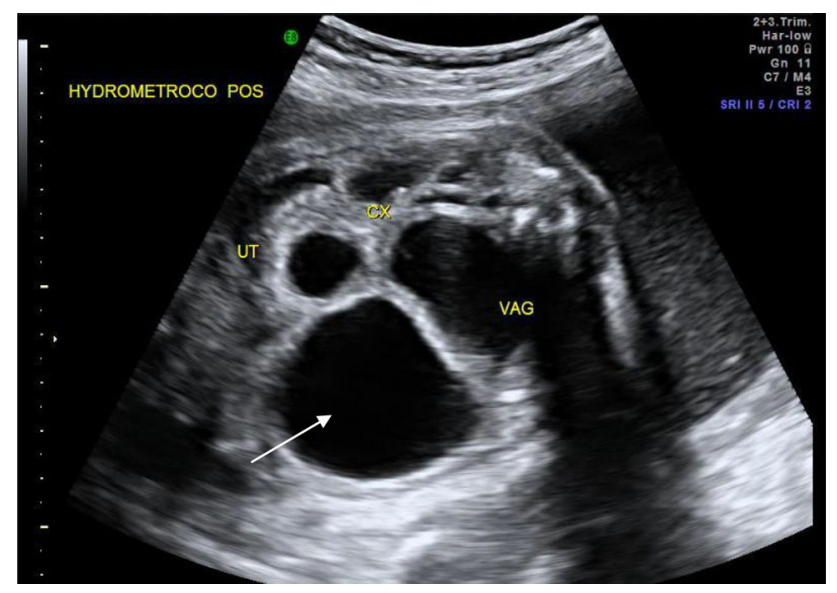

Fig. 4 A parasagittal section of fetal abdomen showing a distended vagina and uterine cavity posterior to the bladder (arrow). CX, cervix; UT, uterus; VAG, vagina

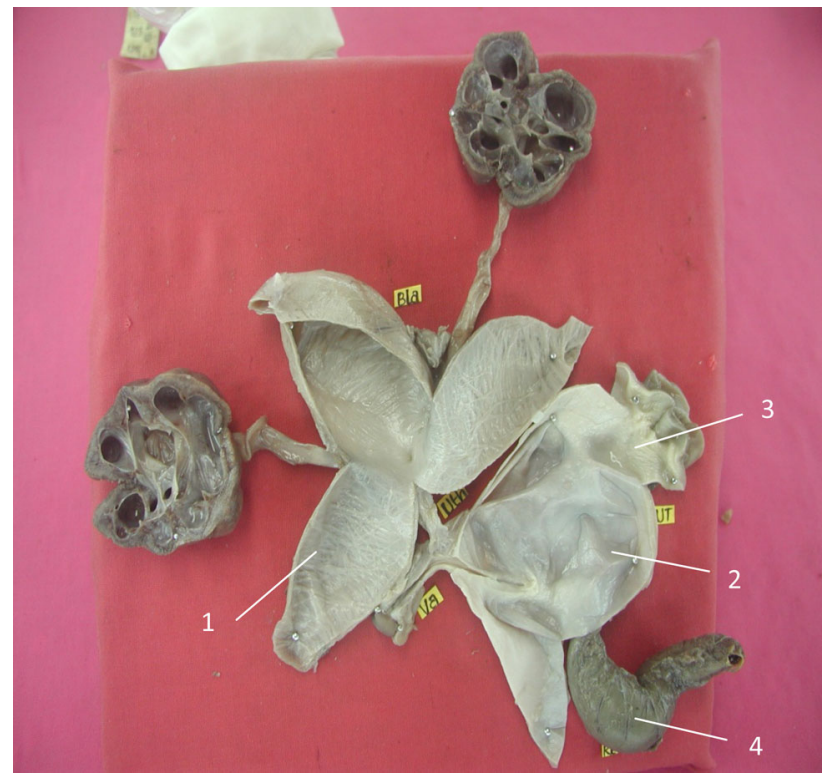

Fig. 5 Cut section of distended bladder (1), vagina (2) and uterine (3) cavity with high anorectal anomaly (4)

autopsy. At autopsy, there was moderate to large amount of blood-stained fluid in the peritoneal, pleural, and pericardial cavities. The lungs, liver, and thymus were hypoplastic. There was hydrometrocolpos with vaginal atresia. There was bilateral pelviectasis, imperforate anus with high anorectal anomaly, and a colovaginal fistula and ambiguous genitalia (Fig. 6).

\section{Case IV}

The fetus of a primigravida with an antenatal ultrasound scan diagnosis of polyhydramnios and a possible intestinal abnormality was sent for autopsy. At autopsy, the fetus was

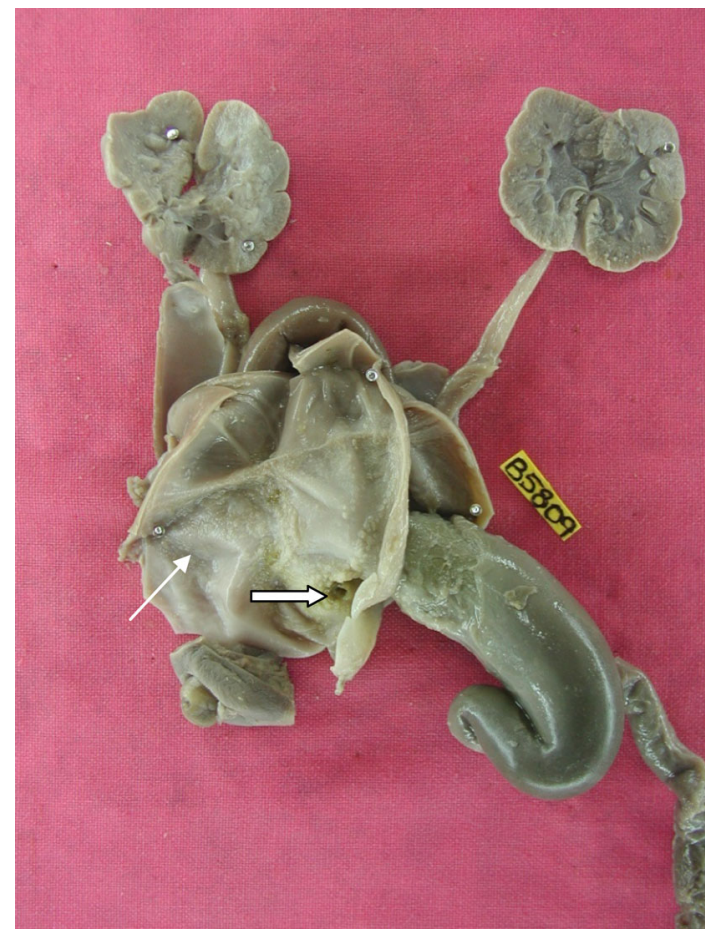

Fig. 6 Cut section of distended vagina (thin arrow) showing a colovaginal fistula (thick arrow) with meconium staining at the fistulous opening

assessed to be at 25-26 weeks of gestation with dysmorphic facies. The arms, forearms, and lower limbs were thin with reduced muscle mass, the abdomen was scaphoid and there was bilateral arachnodactyly. There was hydrocolpos with vaginal atresia (Fig. 7) and the labia and clitoris were hypoplastic. The anus was perforate and anteriorly placed. There was bilateral diaphragmatic eventration and bilateral hypoplastic lungs with pleural effusion (Fig. 8).

Two of the four cases of hydrocolpos/hydrometrocolpos were diagnosed antenatally, one at 27 weeks and the other at 31 weeks. In three of the four cases, the hydrometrocolpos was secondary to atresia of the lower part of the vagina. The fourth case was due to a transverse vaginal septum. In all cases, there were associated abnormalities in other organs. Most notable of these was the presence of pulmonary hypoplasia in all four cases. The kidneys were abnormal in three of the four cases, two were due to bilateral hydronephrosis and the third had unilateral renal agenesis. The external genitalia were ambiguous in all four cases. The anus was imperforate in three cases with a high anorectal anomaly in all three cases and a colovaginal fistula in one. The bladder was distended in all four cases with urethral atresia in one case. There were vertebral/ skeletal abnormalities in all four cases with sacral dysgenesis in two cases, sacral hemivertebra in one case and bilateral arachnodactyly in the fourth. The heart was normal in all cases but there was aberrant origin of the left 


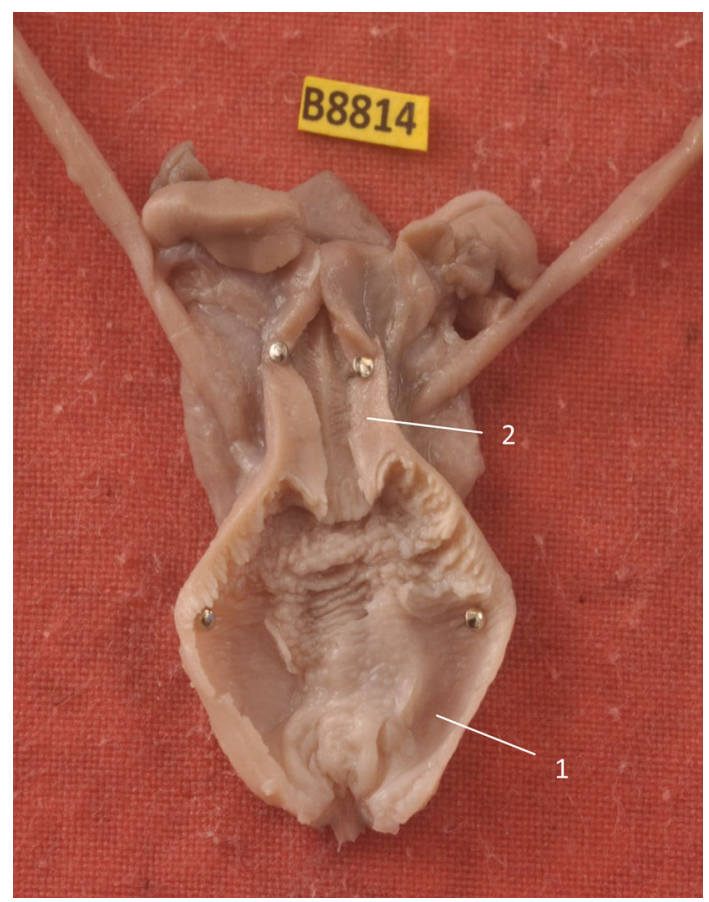

Fig. 7 Cut section of hydrocolpos. Vagina (1); Uterus (2)

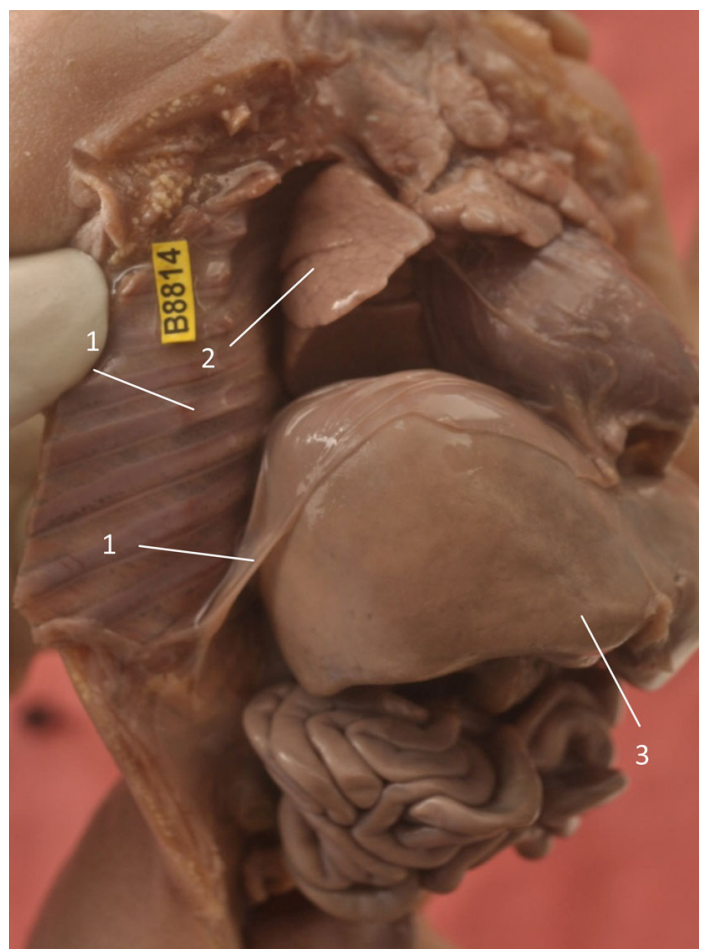

Fig. 8 Autopsy image showing diaphragmatic eventration and hypoplastic lungs. Diaphragm (1); Lung (2); Liver (3)

external carotid artery from the aortic arch in one case. There was diaphragmatic eventration in one case and there was a single umbilical artery in one case (Fig. 9).

\section{Discussion}

Hydrometrocolpos can be isolated or associated with other abnormalities. It can be a part of several syndromes such as McKusick-Kaufman, Ellis-van Creveld, and Bardet-Biedl syndromes [5]. The presentation of hydrometrocolpos is variable. It can present prenatally, at birth, or at any time up to puberty. In the fetus, it typically presents on ultrasound as a cystic mass posterior to the bladder. Prenatal diagnosis of hydrometrocolpos can be difficult as a result of its rarity, variable presentation, and poor ultrasonography. The earliest gestation at diagnosis is reported at 25 weeks and most cases are diagnosed in the third trimester [5]. In the present study, two of the four cases were diagnosed prenatally, the first at 27 weeks and the second at 31 weeks of gestation. Magnetic resonance imaging (MRI) can be a valuable tool in assisting the prenatal diagnosis of fetal hydrometrocolpos [6]. At birth, hydrometrocolpos presents as an abdominal mass or at puberty, when it is usually secondary to an imperforate hymen, as a bulge with a bluish discoloration at the vaginal introitus [7].

The mass in hydrometrocolpos arises secondary to vaginal outlet obstruction which results in retained vaginal and cervical secretions, that are secreted in response to circulating maternal estrogens [2]. Also, associated urinary and intestinal fistulae are usually present leading to urine and meconium being retained [8].

The prenatal differential diagnosis of hydrometrocolpos includes renal lesions, enteric cysts, ovarian cysts, mesenteric cysts, dilated ureter, loops of bowel, anterior sacral meningocoele, pelvic component of sacrococcygeal teratoma, and retroperitoneal cystic lymphatic malformations [9].

Hydrometrocolpos may be secondary to varying degrees of vaginal atresia, a transverse vaginal septum, imperforate hymen, persistent urogenital sinus, or a persistent cloacal anomaly. Certain abnormalities associated with hydrometrocolpos, such as hydronephrosis and pulmonary hypoplasia, which can be seen even prenatally, and renal obstruction, intestinal obstruction, respiratory distress, and leg swelling, as seen in the neonatal period, are a result of secondary manifestations from the pressure of the mass on the surrounding structures. Ambiguous genitalia $[2,10]$, imperforate anus, and vertebral/skeletal abnormalities arise as primary developmental abnormalities. In the present study, the presentation of the fourth case was suggestive of a chromosomal abnormality, whereas in each of the first three cases, there were abnormalities of the genital tract, renal/urinary system, lower gastrointestinal tract, and axial skeleton. This nonrandom association of abnormalities of these systems 
Fig. 9 Chart depicting the frequency of occurrence of other system abnormalities in association with hydrometrocolpos in an autopsy series of four cases at a tertiary referral center between 2009 and 2014

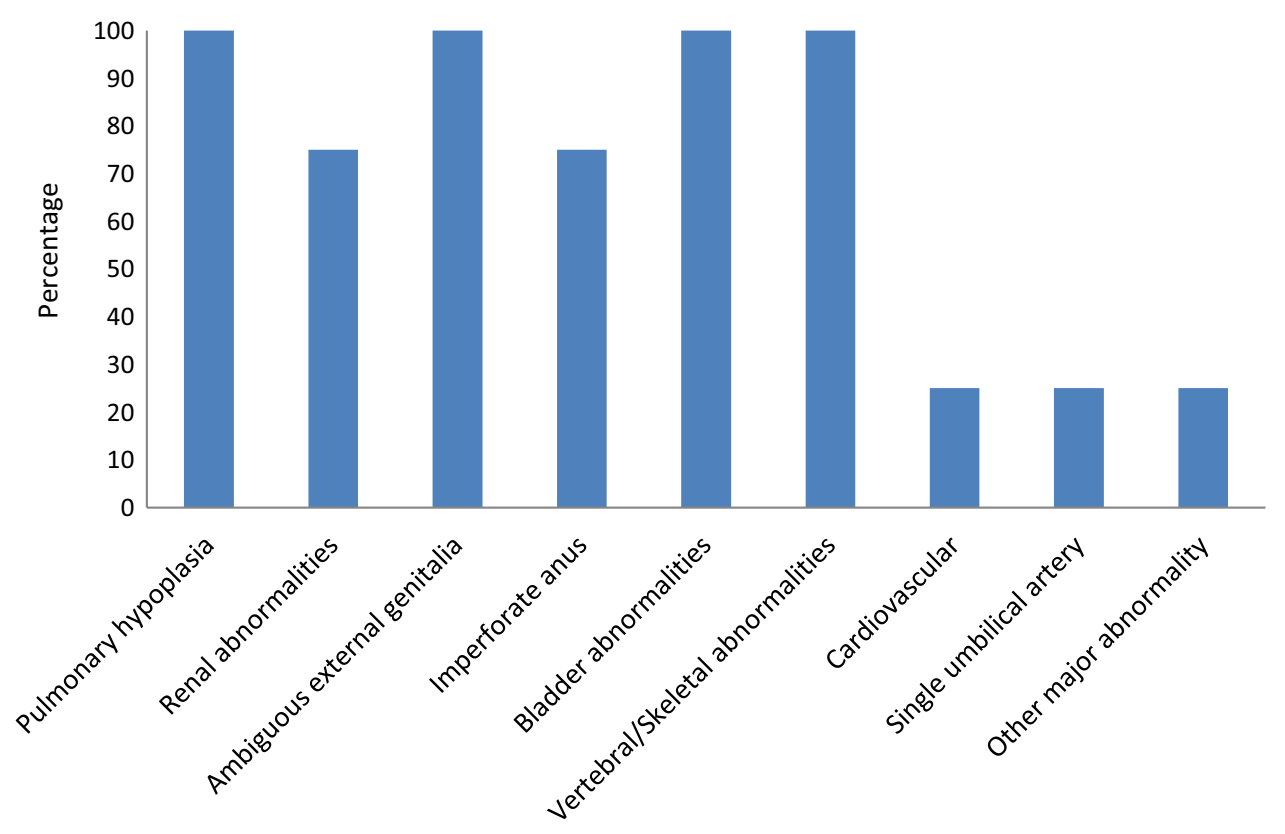

is suggestive of a condition known as lower mesodermal defects sequence [11].

Lower mesodermal defects sequence, as described by Pauli [11], involves the recurrent clustering of abnormalities of the renal, urinary, genital, lower gastrointestinal, and axial skeletal systems. All these structures derive from the lower infraumbilical portion of the intraembryonic mesoderm. The pattern of abnormalities encountered includes kidneys (absence, hypoplasia, or malmigration), ureters (absence or stenosis), bladder (absent, small, or markedly enlarged), urethra (absent or atretic), vagina (hydrometrocolpos), internal genitalia (abnormalities of the uterus and fallopian tubes), lumbosacral vertebra (hemivertebra and sacral agenesis/dysgenesis), adrenals, gonads, imperforate anus, and ambiguous genitalia. Not all abnormalities need to be present in all cases. But all structures involved share a common embryologic origin. Occasionally, structures topologically distant may be abnormal, such as upper or lower limb, cardiac, pulmonary, and tracheoesophageal defects [12].

Lower mesodermal defects sequence appears to arise either as a result of a primary developmental failure or a secondary disruption of normally formed tissues (around the third or fourth week after conception). No specific environmental insult has been identified. Lower mesodermal defects sequence had been identified as an underdiagnosed cause of stillbirth and early neonatal death. Ascertaining the diagnosis is helpful in counseling the bereaving parents. Firstly, it is the result of a single abnormality, namely, abnormality in the development of the lower embryonic mesoderm rather than there being multiple 'abnormalities' in the fetus. Secondly, no specific environmental insult has been identified, which means that the condition is not due to something the parents might have done or not done. Lastly, the diagnosis implies little or no risk of recurrence, which helps in reassuring the parents.

\section{Conclusion}

Hydrometrocolpos may be an isolated condition or associated with other abnormalities. When isolated, the prognosis is excellent. Associated abnormalities may be primary developmental abnormalities or secondary to pressure from the mass. Lower mesodermal defects sequence is a recurrent clustering of specific anomalies related to structures having a common embryological origin. The diagnosis of the condition helps in prognostication and counseling the parents.

Compliance with Ethical Standards

Conflict of interest None.

\section{References}

1. Ayaz UA, Dilli A, Api A. Ultrasonographic diagnosis of congenital hydrometrocolpos in prenatal and newborn period: a case report. Med Ultrason. 2011;13(3):234-6.

2. Nunes E, Branco M, Meyer F, et al. Prenatal diagnosis of fetal hydrometrocolpos. Acta Obstet Ginecol Port. 2014;8(4):405-7.

3. Saxena R, Divan G, Long AM, et al. Fetal hydrometrocolpos. Ultrasound Obstet Gynecol. 1993;3:360-1.

4. Hayashi S, Sago H, Kashima K, et al. Prenatal diagnosis of fetal hydrometrocolpos secondary to a cloacal anomaly by magnetic resonance imaging. Ultrasound Obstet Gynecol. 2005;26:577-9. 
5. Celayir AC, Kurt G, Sahin C, et al. Spectrum of etiologies causing hydrometrocolpos. J Neonat Surg. 2013;2(1):5.

6. Hung $\mathrm{YH}$, Tsai $\mathrm{CC}, \mathrm{Ou} \mathrm{CY}$, et al. Late prenatal diagnosis of hydrometrocolpos secondary to a cloacal anomaly by abdominal ultrasonography with complementary magnetic resonance imaging. Taiwan J Obstet Gynecol. 2008;47(1):79-83.

7. Vitale V, Cigliano B, Vallone G. Imperforate hymen causing congenital hydrometrocolpos. J Ultrasound. 2013;16(1):37-9.

8. Troyano-Luque JM, Alvarez de la Rosa M, Padilla AI, et al. Preand postnatal features of hydrometrocolpos in one fetus of a dizygotic twin pregnancy. DSJUOG. 2009;3(3):97-101.
9. Taori K, Krishnan V, Sharbidre KG, et al. Prenatal sonographic diagnosis of fetal persistent urogenital sinus with congenital hydrocolpos. Ultrasound Obstet Gynecol. 2010;36:641-3.

10. Gilboa Y, Lipitz S, Zalel Y, et al. Prenatal diagnosis of ascites with hydrometrocolpos and uterus didelphys in a monoamniotic twin pregnancy. J Ultrasound Med. 2007;26:373-6.

11. Pauli RM. Lower mesodermal defects: a common cause of fetal and early neonatal death. Am J Med Genet. 1994;50(2):154-72.

12. Tos T, Aktas S, Ikbal M, et al. A case of lower mesodermal defects sequence. Genet Couns. 2010;21(4):381-4. 